\title{
Aproximaciones a la estructura institucional de algunos procesos de integración
}

\author{
ÁNGELA GÓMEZ JUTINICO \\ Fecha de recepción: 26-06-2009 - Aprobación: 21 -07-2009
}

\section{Resumen}

A través de los años se han creado y consolidado diversos organismos internacionales de unión económica, como son la Comunidad Andina de Naciones (CAN), el Mercosur y la Unión Económica Europea. Dichas uniones fueron creadas con el propósito principal de llevar a cabo la integración económica y comercial de los diversos países que las componen, estableciendo mercados comunes.

Para alcanzar su objetivo, cada una de esas organizaciones ha venido creando internamente una serie de órganos e instituciones, bajo una óptica de división clara del trabajo jerárquica y funcionalmente establecida. Así, se encuentra que en la CAN, el Mercosur y la Unión Europea existen diversos órganos que cumplen cada uno unas funciones determinadas, de orden Legislativo, Judicial y Ejecutivo. De igual forma, se crean órganos de carácter netamente consultivo, y, en algunas de ellas, otros instaurados como entidades de apoyo financiero y monetario a los países miembros.

Sin embargo, a pesar de estas similitudes, existen diferencias en las estructuras internas, principalmente en lo tocante con la distribución de los poderes y de las funciones de cada una de las instituciones.

En todo caso, el presente artículo se propone establecer, como un avance en la investigación, las similitudes y diferencias existentes entre las uniones económicas, para establecer si comparten la misma estructura, la comparten parcialmente o definitivamente no tiene nada en común.

\section{Palabras clave}

Unión económica, mercado común, división del trabajo, órganos e instituciones.

\section{Abstract}

Through theyears, different international organizations of economic union, such as The Andean Nations Community (CAN in Spanish), Mercosur, and the European Union have been created and consolidated. These associations were created mainly in order to carry out the economic and commercial integration of the different countries that are part of them, establishing common markets.

To insure such purpose, the different organizations have been internally creating a series of agencies and institutions, under a clear hierarchical division that is functionally established. It can be appreciated in the CAN, the Mercosur, and the European Union because they have diverse agencies that fulfill determined assignments of legislative, legal, and executive order. In addition, there are agencies created only for the purpose of consulting and in some of them others that give financial and monetary support to the countries that are part of them.

Even though there are similar structures, every institution has differences in the inner distribution of power and functions.

This article aims to establish, as a step forward in the investigation, the similarities and differences between the economic unions mentioned in order to establish whether they share the same structure partially or if they are absolutely different.

\section{Key words}

Economic union, common market, job division, agencies and institutions. 


\section{ÁNGELA GÓMEZ JUTINICO}

Abogada de la Universidad Libre de Colombia, especialista en Derecho Comercial de la Pontificia Universidad Javeriana, candidata a máster en Derecho Económico de la Pontificia Universidad Javeriana. Profesora investigadora del Politécnico Grancolombiano. anggomez@poligran.edu.co

\section{Metodología}

Es preciso señalar que el presente texto corresponde a un avance obtenido como fruto del desarrollo de un proyecto de investigación que se encuentra adscrito a la línea de investigación en Derecho Económico, del Programa de Derecho del Politécnico Grancolombiano.

\section{Avance}

\section{INTRODUCCIÓN}

Todos los organismos, nacionales e internacionales, crean órganos e instituciones para el cumplimiento efectivo de los fines para los cuales fueron creados, mediante una división clara del trabajo jerárquica y funcionalmente establecida, de modo tal que, mientras institucionalmente se crean redes cada vez más grandes y complejas, en el nivel de funcionamiento se simplifican las labores y trámites ante dichos organismos para la realización de sus objetivos principales.

Es así como sucede con las principales uniones económicas mundiales, como son la Comunidad Andina de Naciones, el Mercosur y la Unión Europea, todas creadas con el fin primordial de llevar a feliz término el proceso de integración regional y comercial en sus respectivas áreas de actividad, estableciendo mercados comunes.

En ese contexto, se hace imperiosa la necesidad de determinar cómo es la estructura institucional de cada uno de los esos organismos económicos supranacionales, poniendo de relieve sus diferencias y similitudes en materia de instituciones y órganos que los integran, para así establecer si comparten la misma estructura, si la comparten parcialmente o definitivamente no tienen nada en común.

\footnotetext{
1 En http://www.comunidadandina.org/sai/que.htm.
}

\section{Comunidad Andina de Naciones}

El conjunto de instituciones de la Comunidad Andina de Naciones (CAN) se encuentra integrado en el sistema andino de integración (SAI), cuya finalidad es "permitir la coordinación efectiva entre sí para profundizar la integración subregional andina, promover su proyección externa y robustecer las acciones relacionadas con el proceso de integración" ${ }^{1}$. Dichas instituciones se rigen por el Acuerdo de Cartagena, que es el que les dio origen, por sus respectivos tratados constitutivos y por los protocolos modificatorios, y son las que a continuación se enuncian.

a. Como órganos de dirección y de decisión:

- Consejo Presidencial

- Consejo Andino de Ministros de Relaciones Exteriores

- Comisión de la Comunidad Andina.

b. Como órganos que detentan las tres ramas del poder:

- Secretaría General (Ejecutivo)

- Parlamento Andino (Legislativo)

- Tribunal Andino de Justicia (Jurisdiccional).

c. Como instituciones consultivas:

- Consejo Consultivo Empresarial

- Consejo Consultivo Laboral

- Consejo Consultivo de los Pueblos Indígenas.

d. Cómo instituciones financieras:

- Corporación Andina de Fomento

- Fondo Latinoamericano de Reservas.

e. Como instancias sociales:

- Convenio Hipólito Unanue

- Convenio Simón Rodríguez

- Universidad Andina Simón Bolívar.

\section{Consejo Presidencial}

Es el máximo órgano del SAI, cuya principal labor es dirigir el proceso de integración subregional andina, estableciendo los lineamientos generales para llevar a cabo dicho proceso mediante directrices y mandatos. 
Está integrado por los jefes de Estado de los Estados parte (Colombia, Bolivia, Perú y Ecuador).

Sus funciones principales son:

a. Definir la política de integración, haciéndole seguimiento y evaluando sus resultados.

b. Coordinar la labor de los diferentes órganos e instituciones del SAI, orientando e impulsando sus acciones relativas a la integración y revisando y pronunciándose acerca de sus iniciativas, informes y recomendaciones.

\section{Consejo Andino de Ministros de Relacio- nes Exteriores}

Es el órgano de dirección política, cuya función principal es verificar el cumplimiento de los objetivos integracionistas y formular y ejecutar la política exterior de la CAN, materia en la cual tiene competencia legislativa, mediante declaraciones y decisiones. Está presidido por el ministro de Relaciones Exteriores del país que tiene la presidencia del Consejo Presidencial e integrado por los cancilleres de los Estados parte.

Sus funciones principales, entre otras, son:

a. Formular la política exterior de los Estados parte, orientando y coordinando las acciones externas de todos los órganos e instituciones del sal.

b. Cumplir las directrices del Consejo Presidencial y velar por el cumplimiento de aquellas dirigidas a los otros órganos andinos.

c. Representar a la cAN en asuntos y actos de interés común.

d. Formular, ejecutar y evaluar, junto con la Comisión, la política general de integración subregional.

\section{Comisión de la Comunidad Andina}

Es el órgano encargado de formular, ejecutar y evaluar la política de integración subregional andina en materia de comercio e inversiones. Comparte las funciones del Consejo de Ministros de Relaciones Exteriores en cuanto a la adopción de las medidas necesarias para el cumplimiento de los objetivos integracionistas, el cumplimiento de las directrices del Consejo Presidencial y la labor de coordinación de los países miembros en foros y negociaciones internacionales.

\subsection{Secretaría General}

Es el órgano ejecutivo del SAI, cuya función principal es administrar el proceso de integración subregional. Está dirigida por un secretario general elegido por consenso en el Consejo de Ministros de Relaciones Exteriores. Tiene función propositiva, toda vez que puede formular propuestas de decisión ante los órganos de decisión y dirección. Entre otras de sus funciones están:

a. Decidir sobre los asuntos puestos a su conocimiento.

b. Velar por el cumplimiento de los compromisos adquiridos por la CAN.

c. Mantener vínculos con los países miembros y con los órganos ejecutivos de otras organizaciones regionales de integración y cooperación.

\section{Parlamento Andino}

Es el órgano deliberante del SAI, el cual representa a todos los pueblos de la Comunidad. Sus representantes son elegidos por los congresos o parlamentos nacionales de cada uno de los Estados miembros, aunque en virtud del Protocolo adicional modificatorio del Tratado constitutivo, la elección de los representantes se hará por voto directo y universal. Entre sus funciones principales e encuentran:

a. Participar en la generación normativa del proceso, mediante sugerencias a los órganos de dirección del SAI.

b. Promover la armonización de las legislaciones de los Estados miembros.

c. Fomentar las labores de cooperación y coordinación entre los parlamentos de los Estados miembros y de terceros países.

\section{Tribunal Andino de Justicia}

Es el órgano jurisdiccional del sAI, integrado por cuatro magistrados, representantes de cada uno de los países miembros, cuyas funciones principales son: 
a. Controlar la legalidad de las normas comunitarias, mediante la acción de nulidad.

b. Interpretar las normas del ordenamiento jurídico andino, velando por la aplicación uniforme en el territorio de su jurisdicción.

c. Dirimir las controversias.

d. Conocer del recurso por omisión o inactividad.

e. Tiene función arbitral.

f. Tiene jurisdicción laboral.

\section{Consejo Consultivo Empresarial}

Es un órgano consultivo del sector empresarial, creado en la búsqueda de "una mayor participación del sector empresarial en la construcción del proceso de integración tendiente a conformar el mercado común" (Comunidad Andina de Naciones).

Está integrado por cuatro delegados elegidos entre los directivos más importantes de organizaciones empresariales representativas de los Estados parte. Su función principal es emitir conceptos ante el Consejo de Ministros Exteriores, ante la Comisión Andina y ante la Secretaría General, a petición de ellos o de oficio, sobre temas de su interés y competencia.

\section{Consejo Consultivo Laboral}

Es un órgano consultivo del SAI, creado en la búsqueda de una mayor participación del sector laboral en el proceso de integración subregional.

Está integrado por cuatro delegado de las organizaciones laborales más representativas de los países miembros. Su función principal es emitir concepto ante el Consejo de Ministros de Relaciones Exteriores, la Comisión Andina y la Secretaría General, a petición de ellos o de oficio, sobre temas de su interés y competencia.

\section{Consejo Consultivo de los Puebles Indí- genas}

Es un órgano consultivo del SAI, creado en la búsqueda de una mayor participación de las comunidades indígenas.

Está integrado por un representante y un suplente de las organizaciones indígenas más importantes de cada uno de los países miembros, además de un representante, en calidad de observador, del Fondo para el Desarrollo de los Pueblos Indígenas de América Latina y del Caribe, Coordinadora de las Organizaciones Indígenas de la Cuenca Amazónica, Coordinadora Andina de las Organizaciones Indígenas y Enlace Continental de Mujeres Indígenas de Suramérica.

Su función principal es emitir conceptos ante el Consejo de Ministros de Relaciones Exteriores, la Comisión Andina y la Secretaría General, a petición de ellos o de oficio, además de compartir y difundir experiencias, fortaleciendo de tal suerte los vínculos de cooperación entre las organizaciones indígenas, los gobiernos y la sociedad civil.

\section{Corporación Andina de Fomento (CAF)}

Órgano financiero que actualmente constituye la principal fuente y movilización de recursos hacía la Comunidad Andina. Se trata de una institución financiera multilateral, cuyo principal objetivo es velar por el desarrollo sostenible de los países accionistas y la integración subregional. Su ayuda está dirigida tanto a los sectores público y privado, ofreciendo una gama de productos y servicios a los gobiernos de los países, instituciones financieras y empresas públicas y privadas. Combina los objetivos financieros, con proyectos ambientales y de desarrollo social.

\section{Fondo Latinoamericano de Reservas (FLAR)}

Institución financiera del SAI cuyo objetivo principal es brindar apoyo a las balanzas de pagos de los países miembros, otorgando créditos a ellos mismos o a terceros.

Entre sus principales funciones se encuentran:

a. Armonizar las políticas monetarias, cambiarias y financieras de los países.

b. Mejorar las condiciones de inversiones de reservas internacionales efectuadas por las naciones andinas.

\section{Convenio Hipólito Unane (CAN)}

Institución del SAI, cuyo objetivo principal es coordinar y apoyar los esfuerzos de los países miembros en materia de salud. En ese sentido coopera con otros órganos subregionales, regionales e internacionales. 


\section{Convenio Simón Rodríguez}

Órgano del sal, cuya principal función en fungir como un foro de debate, participación y coordinación en los temas de las áreas sociales y laborales de la can, en especial en lo relativo a políticas referentes al fomento del empleo, la formación y capacitación laboral, la seguridad y salud en el trabajo, la seguridad social y las migraciones laborales.

Esta institución está constituida por los siguientes órganos: la Conferencia, máximo órgano, que adopta las decisiones por consenso; las comisiones especializadas de trabajo, cuya labor es de asesoría principalmente, y la Secretaría Técnica.

\section{Universidad Simón Bolívar}

Institución del sai cuyos objetivos principales son la investigación, la enseñanza, la formación posuniversitaria y la prestación de servicios y el fomento de la cooperación entre las diversas universidades de la subregión.

\section{Mercado Común del Sur (Mercosur)}

Por su parte el Mercosur se divide internamente en órganos principales y órganos de carácter auxiliar. Los primeros son aquellos que han sido establecidos en los tratados funcionales del organismo y son de carácter permanente; los segundos son los órganos que dependen de los principales, a quienes elevan sus propuestas. Se crean para llevar a cabo el proceso de integración y su funcionamiento es eventual, dependiendo del "mantenimiento de los fines para los que fueron creados" (Ferreira, 2004).

Entre los órganos principales se encuentran:

- Consejo de Mercado Común

- Grupo de Mercado Común

- Comisión de Comercio de Mercosur

- Parlamento de Mercosur

- Foro Consultivo, Económico y Social

- Comisión de Representantes Permanentes del Mercosur
- Secretaría Administrativa

- Tribunal Permanente de Revisión.

\section{Consejo de Mercado Común (смC):}

Es el máximo órgano, que tiene entre sus principales funciones la de tomar las decisiones relativas al proceso de integración y liderar dicho proceso. Ejerce la personalidad jurídica del Mercosur.

Está integrado por los ministros de Relaciones Exteriores y los de Economía, o sus equivalentes, de los países miembros, quienes sesionan por una vez al semestre con los jefes de Estado.

Fue creado por el Tratado de Asunción, pero adopta sus funciones y estructura del Protocolo de Ouro Preto.

Las funciones principales del Consejo, entre otras, son:

a. Velar por el cumplimiento del Tratado de Asunción y demás acuerdos firmados en su marco.

b. Liderar el proceso integracionista, mediante la formulación de políticas y acciones necesarias.

c. Negociar y firmar acuerdos en nombre del Mercosur.

d. Pronunciarse sobre las propuestas del Grupo Común.

\section{Grupo de Mercado Común (Gмc)}

Órgano ejecutivo del Mercosur, cuya finalidad principal es velar por el cumplimiento de las decisiones del máximo órgano.

Está integrado por cuatro titulares y cuatro alternos representantes del Ministerio de Relaciones Exteriores, Ministerio de Economía y los bancos centrales de cada país miembro. En su ámbito funcionan los subgrupos de trabajo, para las grandes áreas de trabajo, y las reuniones especializadas, para áreas más específicas.

Sus principales funciones, entre otras, son:

a. Formular recomendaciones para la ejecución del Tratado de Asunción y otros acuerdos.

b. Evaluar las propuestas de los Estados parte relativas al Mercosur. 
c. Mantener relaciones con el Parlamento de Mercosur.

d. Participar en la solución de controversias en las condiciones establecidas en el Protocolo adicional para la solución de controversias del Tratado de Asunción.

\section{Comisión de Comercio del Mercosur (сCM)}

Órgano aduanero y comercial, encargado de hacerle seguimiento al proceso de creación de la unión aduanera. Está integrada por cuatro titulares y cuatro alternos de cada Estado parte, y coordinada por los ministros de Relaciones Exteriores.

Asiste al Grupo de Mercado Común, pero con capacidad decisoria propia. Fue creado por la Decisión 9/94 del consejo máximo, y formalizado por el Protocolo de Ouro Preto.

Trabaja en comités técnicos, frente a grandes áreas de trabajo. Sus funciones esenciales son:

a. Analizar la evolución de la política comercial común para el funcionamiento de la unión aduanera, formulando las respectivas propuestas ante el GMC.

b. Hacer seguimiento de los instrumentos de política comercial de los Estados parte.

\section{Parlamento de Mercosur}

Antigua Comisión Parlamentaria Conjunta, es el órgano de representación de los pueblos, que funge de órgano consultivo deliberativo y de formulación de propuestas. Está compuesto por 64 miembros, 16 por cada Estado miembro, elegidos por los parlamentos de cada país, pero que posteriormente lo serán por voto directo y simultáneo.

Sus principales funciones son:

1. Colaborar y acelerar la armonización de las legislaciones de los Estados parte y de los procedimientos internos necesarios para materializar la normatividad que integran el ordenamiento jurídico del Mercosur.

2. Elaborar una política legislativa de integración.
3. Mantener relaciones con los parlamentos de terceros países y de otras organizaciones internacionales.

\section{Foro Consultivo Económico y Social (FCES)}

Es el órgano de representación de los sectores económico y social de los Estados miembros, en el que no participan los gobiernos. Tiene una función consultiva, mas no obligante, especialmente en los temas económicos y sociales, sobre los cuales puede emitir concepto a solicitud de otros órganos o de oficio.

Está integrado por los representantes de organizaciones de trabajadores y empleadores, cooperativas, medios de comunicación, profesionales liberales, consumidores, medioambientalistas, pequeñas, medianas y grandes empresas (Resolución GMC 35/00).

Está conformada por las secciones nacionales, con autonomía organizativa y que representan diversos sectores. Su órgano principal es el Plenario del Foro.

Sus principales funciones son:

a. Cooperar para promover progreso económico y social.

b. Analizar los impactos económicos y sociales derivados del proceso de integración.

c. Proponer normas y políticas sociales y económicas.

d. Realizar investigaciones y estudios.

e. Propender por una mayor participación de la sociedad civil en la vida del Mercosur.

\section{Comisión de Representantes Permanen- tes del Mercosur (CRPM)}

Es un órgano de gestión política y representación permanente del Mercosur ante terceros (Decisión 11/03). Está integrada por un representante de cada país miembro y por un presidente elegido por el CMC.

Tiene como principales funciones las siguientes:

a. Propender un ámbito permanente de negociación y gestión política.

b. Afianzar las relaciones económicas, sociales y parlamentarias del Mercosur, estableciendo vínculos 
con el Parlamento, el Foro Consultivo Económico y Social y con las reuniones especializadas.

\section{Secretaría Administrativa del Mercosur (SAM)}

Es el órgano de apoyo operativo, cuya función esencial es prestar servicios a los demás órganos del Mercosur, especialmente en lo relacionado con todo lo que requiera publicidad oficial. Es una institución de carácter administrativo.

Entre sus principales funciones se encuentran:

a. Hacer el archivo oficial del Mercosur.

b. Publicar y difundir las normas adoptadas en el marco de la organización internacional.

c. Coordinar las reuniones de los órganos decisorios y de las demás instituciones del Mercosur, en lo posible.

d. Servir de centro de información para los Estados parte.

e. Realizar las labores encomendadas por el CMC, GMC y CCM.

f. Elaborar un proyecto de presupuesto y ejecutarlo correctamente, una vez haya sido aprobado por el GMC.

g. Rendir cuentas al CMc y presentar informe sobre las actividades.

\section{Tribunal Permanente de Revisión (Pro- tocolo de Olivos, 2002)}

Órgano judicial del Mercosur, cuya función además de ser jurisdiccional es consultiva. Conoce de los casos presentados contra alguno de los Estados miembros, tanto por otro Estado como por un particular.

Está integrado por cinco árbitros, de los cuales cuatro son elegidos directamente por los Estados parte, y uno es designado por común acuerdo.

Entre sus principales funciones se encuentra:
a. Dictar medidas provisionales.
b. Actuar como tribunal de revisión.

c. Actuar como tribunal de única instancia, de común acuerdo por las partes.

d. Dar opiniones consultivas.

La creación de órganos auxiliares del Mercosur depende del CMC, del GMC y de la CCM, cuyas funciones se desarrollan en el marco laboral, de desarrollo social y servicios, entre otras.

\section{Unión Europea}

La Unión Europea, por su parte, está constituida por los siguientes órganos o instituciones, vigentes para los 27 países que hacen parte de este organismo comunitario internacional:

- Consejo Europeo

- Comisión Europea

- Parlamento Europeo

- Tribunal de Justicia Europeo

- Órganos consultivos

- Órganos financieros

- Órganos interinstitucionales

- Órganos especializados

\subsection{Consejo Europeo}

Reúne a los jefes de Estado y al presidente de la Comisión. Su principal función es definir las orientaciones y prioridades políticas generales de la Unión, además de impulsar su desarrollo.

El Consejo se divide en diversas formaciones, dependiendo de los temas que se traten y del ámbito de su aplicación, representadas por los ministros y comisarios correspondientes de cada país. Sin embargo, las decisiones que se toman internamente en cada una de las formaciones son "únicas", ya que son expedidas a nombre del Consejo Europeo, independientemente de la formación que la haya tomado.

Así, el Consejo se divide en:

a. Asuntos generales y de relaciones exteriores.

b. Política exterior y seguridad común. 

c. Asuntos económicos y financieros.
d. Justicia y asuntos del interior.
e. Empleo, política social, sanidad y consumidores.
f. Competitividad.
g. Transporte, telecomunicaciones y energía.
h. Agricultura y pesca.
i. Medioambiente.
j. Educación, juventud y cultura.

\section{Comisión Europea}

Participa en el proceso decisorio de la Unión Europea. Personifica el interés general de la Unión.

Entre sus funciones principales se encuentra:

a. Formular propuestas de leyes al Parlamento y al Consejo.

b. Administrar y ejecutar la política de la Unión.

c. Junto con el Tribunal, aplicar y velar por el cumplimiento del Derecho comunitario.

d. Negociar acuerdos internacionales, en especial sobre comercio y cooperación.

\section{Parlamento Europeo}

Elegido por sufragio directo, representa los intereses de los ciudadanos de todos los países miembros.

Se encarga de preparar las leyes en todo lo que tenga que ver con la vida en comunidad, ya sea en materia de medio ambiente, derechos de los consumidores, bienes y servicios, entre otras.

Comparte poderes con el Consejo, en lo relacionado con la aprobación del presupuesto anual de la Unión, al igual que tiene competencias de control de las actividades de todos los órganos e instituciones comunitarias, por medio de derechos de petición de los ciudadanos, investigaciones, etcétera.
Está compuesto por:

- Presidente, que representa al Parlamento en el exterior y frente a otras instituciones comunitarias

- Diputados, agrupados por afinidades políticas

- Grupos políticos, también por afinidades políticas y no por nacionalidad

- Comisiones parlamentarias, que junto con los diputados presentan propuestas legislativas

- Delegaciones, que mantienen relaciones con otros parlamentos de terceros países

- Órganos políticos

- Secretaría General.

\section{Tribunal de Justicia}

Compuesto por 27 jueces y 8 abogados generales, designados de común acuerdo entre los gobiernos de los Estados parte. Tiene competencias jurisdiccionales estrictamente definidas, entre ellas la prejudicial y el conocimiento de los recursos.

- Prejudicial: se refiere a cuestiones de interpretación del Derecho comunitario, al igual que la validez de un acto comunitario.

- Recursos: por incumplimiento, por anulación, por omisión, casación y reexamen.

\section{Órganos consultivos}

a. Económico y social: cuyo objetivo principal es crear un puente entre la Unión y la sociedad civil organizada, en todos los niveles, de manera tal que esta última encuentre un espacio abierto de participación activa.

b. De las regiones: tiene la función de presentar los puntos de vista locales y regionales en la legislación de la Unión Europea.

\section{Órganos financieros}

a. Banco Europeo de Inversiones: como fuente de recursos económicos y financieros para el sector público y privado de los Estados miembros. 
b. Fondo de Inversiones: como fuente de recursos económicos y financieros enfocado en la pequeña empresa.

c. Banco Central: cuya principal función es gestionar el euro y preservar la estabilidad de los precios, además de establecer la política económica y monetaria de la Unión Europea.

\section{Órganos interinstitucionales}

a. Oficina de publicaciones: actúa como editorial de las instituciones de la Unión Europea, elaborando y distribuyendo todas las publicaciones oficiales del organismo.

b. Oficina europea de selección de personal: su función es preparar los concursos destinados a la selección y contratación del personal que trabajará en todas las instituciones de la Unión Europea.

c. Escuela Europea de Administración: ofrece formación en distinta áreas para el personal de las instituciones y órganos de la Unión.

\section{Órganos especializados}

a. Defensor del pueblo: establece una intermediación entre los ciudadanos y la Unión, investigando y detectando malas gestiones por parte de sus órganos.

b. Supervisor europeo de protección de datos: busca garantizar la preservación del derecho a la intimidad de las personas en el tratamiento de sus datos personales, frente a las actividades de los órganos de la Unión Europea.

\section{Conclusiones}

Lo que resulta más evidente, y lo que es de esperarse en toda estructura organizada, es que tanto en la Comunidad Andina de Naciones como en el Mercosur y la Unión Europea se ejercen los tres poderes: Ejecutivo, Legislativo y Judicial.

Existen, así, unos órganos de dirección y decisión, máximas instituciones de las estructuras -el Consejo Presidencial en la can, el Consejo de Mercado Común en el Mercosur y el Consejo de la Unión Europea- que básicamente gobiernan los respectivos procesos de integración económica y regional, lo que lleva a pensar que ejercen el poder ejecutivo, auxiliado por otros órganos subalternos, como son el Consejo Andino de Ministros de Relaciones Exteriores para la CAN, el Grupo de Mercado Común para el Mercosur y la Comisión Europea para la Unión Europea.

Además, existen unos órganos inspirados en la idea de un legislador comunitario, como son los respectivos parlamentos de cada una de las comunidades económicas, así como unos órganos jurisdiccionales bajo la figura de los tribunales comunitarios, como se evidencia en las estructuras institucionales objeto de estudio.

Así mismo, es evidente la existencia en dichas estructuras de órganos eminentemente consultivos, sobre temas económicos y sociales, prevalentemente, aspecto en el que al parecer ponen más énfasis en el proceso integracionista.

A pesar de las similitudes aparentes que existen entre los tres organismos internacionales objeto de estudio, la mayor diferencia que existe entre ellos es como realmente se distribuyen los tres poderes. En efecto. Se encuentra que dependiendo del órgano de que se trate, éste ejerce poderes tanto ejecutivos como judiciales, y hasta legislativos, al tal punto que los respectivos parlamentos llegan a tener una facultad meramente consultiva y no decisoria, dejándole el rol de hacer leyes a las instituciones en la punta de la pirámide. Este es el caso de la can y del Mercosur, en los que el Parlamento tiene competencia para formular meras propuestas de leyes, cuya decisión final radica en cabeza de los máximos órganos comunitarios y de sus auxiliares; contrario sensu, en la Unión Europea el Parlamento sí ejerce efectivamente labores de legislador, empero ayudado por los demás órganos de la estructura que tienen facultad para proponer proyectos de leyes.

Otra diferencia importante radica en la existencia de instituciones de apoyo financiero, solamente presentes en la CAN y en la Unión Europea, y por ende ausentes en el Mercosur. Dichos órganos resultan indispensables en el mismo proceso integracionista, al propender por el desarrollo económico sostenido y duradero en todos los frentes de la economía de una nación, tanto el sector público como el sector privado, en grandes, medianas y pequeñas empresas. 


\section{BIBLIOGRAFÍA}

ANDI. En www.andi.org.co.

Arbuet, Vignali, H. (2004). Las claves jurídicas de la integración en los sistemas del Mercosur. y la Unión Europea. Santa Fe: Rubinzal-Culzoni Editores.

Armagangue, J.F. (2007). Estudios sobre el Mercosur. Buenos Aires: Editorial Cuyo

Basombrio, I. (2004). Integración andina: Instituciones y Derecho comunitario. En: Taller Internacional: Integración Regional-Lecciones de la experiencia de la Unión Europea y la Comunidad Andina. (20-21 octubre 2004: Sao Paulo):10-53.

Bustamante, A.M. (2004). Desarrollo institucional de la Comunidad Andina. Aldeamundo, noviembre - abril, (8), 016: 16-23. Universidad de Los Andes, San Cristóbal, Venezuela.

Comunidad Andina de Naciones. Sitio web oficial: www. comunidadandina.org

Deluca, S. et ál. (2003). Unión Europea y Mercosur, los efectos del Derecho comunitario sobre las legislaciones. Buenos Aires: Rubinzal-Culzoni Editores.

Guarnizo Ramírez, J.G. et ál. (s.f). (2007). La política regional en los esquemas de integración suramericanos CAN y Mercosur. En: Papel Político Estudiantil. 3(1):201-230.

Ministerio de Comercio, Industria y Turismo de la República de Colombia. Sitio web oficial: www.mincomercio.gov

Ministerio de Relaciones Exteriores de la República de Colombia. Sitio web oficial: www.cancillería.gov.co

Quindimil J.A. (2006). Instituciones y derecho de la Comunidad Andina ( $1^{\mathrm{a}}$ ed.). Valencia: Editorial Tirant lo Blanch.

Reinoso, A.F. (2000). Relaciones Comunidad Andina Unión Europea y la zona de libre comercio del sur. Lima: Pontificia universidad Católica del Perú, Departamento de Economía.
Rodríguez Álvarez, A.J. (s.f.) Las vidas paralelas de la Comunidad Andina y la Unión Europea. (En línea) www. analítica.com

Suárez Marín, C.A. et ál. (2004). Análisis de la institucionalidad política del Mercosur. Bogotá: Ediciones Ántropos.

Tremolana Álvarez, E. (2006). Los pilares jurídicos de la Comunidad Andina: ¿Elementos decisivos para su supervivencia? Oasis, 12: 317-346. Centro de Investigaciones y Proyectos Especiales (CIPE), Facultad de Finanzas, Gobierno y Relaciones Internacionales, Universidad Externado de Colombia. 